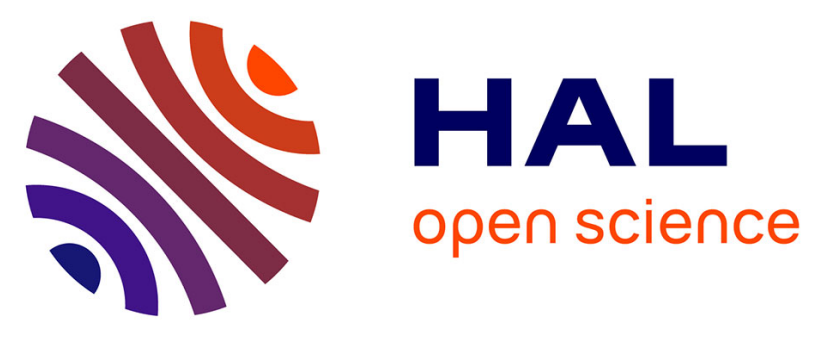

\title{
XEMIS2 Liquid Xenon Compton Camera for Small Animal $3 \gamma$ Medical Imaging: Scintillation Light Measurement
}

Yuwei Zhu, Stephane Acounis, Nicolas Beaupere, Jean-Luc Beney, Julien Bert, Stephane Bouvier, Clotilde Canot, Thomas Carlier, Michel Cherel, Jean-Pierre Cussonneau, et al.

\section{To cite this version:}

Yuwei Zhu, Stephane Acounis, Nicolas Beaupere, Jean-Luc Beney, Julien Bert, et al.. XEMIS2 Liquid Xenon Compton Camera for Small Animal $3 \gamma$ Medical Imaging: Scintillation Light Measurement. 2019 IEEE Nuclear Science Symposium and Medical Imaging Conference (NSS/MIC), Oct 2019, Manchester, United Kingdom. pp.1-4, 10.1109/NSS/MIC42101.2019.9059638 . hal-03044142

HAL Id: hal-03044142

\section{https://imt-atlantique.hal.science/hal-03044142}

Submitted on 15 Dec 2020

HAL is a multi-disciplinary open access archive for the deposit and dissemination of scientific research documents, whether they are published or not. The documents may come from teaching and research institutions in France or abroad, or from public or private research centers.
L'archive ouverte pluridisciplinaire HAL, est destinée au dépôt et à la diffusion de documents scientifiques de niveau recherche, publiés ou non, émanant des établissements d'enseignement et de recherche français ou étrangers, des laboratoires publics ou privés. 


\title{
XEMIS2 Liquid Xenon Compton Camera for Small Animal $3 \gamma$ Medical Imaging: Scintillation Light Measurement
}

\author{
Yuwei Zhu, Stéphane Acounis, Nicolas Beaupère, Jean-Luc Beney, Julien Bert, Stéphane Bouvier, Clotilde Canot, \\ Thomas Carlier, Michel Cherel, Jean-Pierre Cussonneau, Sara Diglio, Debora Giovagnoli, Jérôme Idier, Françoise \\ Kraeber-Bodéré, Patrick Le Ray, Frédéric Lefèvre, Julien Masbou, Eric Morteau, Jean-Sébastien Stutzmann, Dimitris \\ Visvikis, Yajing Xing and Dominique Thers
}

\begin{abstract}
An innovative XEnon Medical Imaging System, named XEMIS2, consisting of a liquid xenon (LXe) Compton camera, is developed to image small animal with $3 \gamma$ imaging technique. The main objective of XEMIS2 involves the 3D localization of a radiopharmaceutical labeled with a specific radionuclide ${ }^{44} \mathrm{Sc}$ and the lessening of the administered radiotracer activity while preserving the image quality in oncology diagnosis. XEMIS2 is a monolithic single-phase detector with a large axial Field Of View (FOV), handling up to nearly $200 \mathrm{~kg}$ of ultra-high-purity liquid xenon. The XEMIS2 facility has been successfully conceived and developed at the SUBATECH laboratory. In XEMIS2, the scintillation signals provide the $\gamma$-rays interaction time. Furthermore, it is possible to pre-localize the $\gamma$-rays interactions spatially and then achieve the virtual fiducialization of the active volume by matching the scintillation signals with the ionization signals. In order to measure scintillation light, we distribute a set of PhotoMultiplier Tubes (PMTs) around the surface of the active area. Besides, a self-triggered scintillation light detection chain has been specially developed for XEMIS2 to carry out continuous data taking with negligible electronics dead-time. It is currently calibrated in the prototype XEMIS1. The calibration results indicate that the dedicated detection chain has a good performance in scintillation light measurement. XEMIS2 is recently under construction in a small animal medical imaging center CIMA, for further preclinical studies.
\end{abstract}

\section{INTRODUCTION}

FOR the past few decades, nuclear medical imaging has extended from organ imaging for tumor localization of a variety of diseases and proven remarkable value in oncology

Manuscript received December 13, 2019. The research presented in this paper has been funded by the E.U., the Region Pays de la Loire in France and by grants from the French National Agency for Research.

Yuwei Zhu is with SUBATECH, IMT Atlantique, CNRS/IN2P3, Université de Nantes, 44307 Nantes, France (corresponding author e-mail: yuwei.zhu@subatech.in2p3.fr).

Stéphane Acounis, Nicolas Beaupère, Jean-Luc Beney, Stéphane Bouvier, Clotilde Canot, Jean-Pierre Cussonneau, Sara Diglio, Patrick Le Ray, Frédéric Lefèvre, Julien Masbou, Eric Morteau, Jean-Sébastien Stutzmann, Yajing Xing and Dominique Thers are with SUBATECH, IMT Atlantique, CNRS/IN2P3, Université de Nantes, 44307 Nantes, France.

Julien Bert, Debora Giovagnoli and Dimitris Visvikis are with INSERM, UMR1101, LaTIM, CHRU Morvan, 29600 Brest, France.

Thomas Carlier and Françoise Kraeber-Bodéré are with Centre Hospitalier Universitaire de Nantes, 44093 Nantes, France.

Michel Cherel is with INSERM U892 équipe 13, 44000 Nantes, France.

Jérôme Idier is with LS2N, Ecole Centrale de Nantes, CNRS/Inp, Université de Nantes, 44307 Nantes, France. diagnosis. For the future improvements of nuclear medical imaging, one principle goal is reducing radiation exposure during the medical exam.

For this purpose, a new low-dose nuclear medical imaging modality, named $3 \gamma$ imaging, has been proposed to preserve the image quality while lessening the administered radiotracer activity significantly in tumor diagnosis. This innovative imaging technique involves two new concepts: a liquid xenon (LXe) Compton telescope and a 3D localization of a new radiotracer labeled with a specific $\left(\beta^{+}, \gamma\right)$ radionuclide, the scandium-44 $\left({ }^{44} \mathrm{Sc}\right)[1]$. The ${ }^{44} \mathrm{Sc}$ can be produced by the ARRONAX cyclotron. Besides, the related study of this radiopharmaceutical is investigated at SUBATECH laboratory, ARRONAX GIP, CRCINA, and Nantes Centre Hospitalier Universitaire (CHU) [2].

The ${ }^{44} \mathrm{Sc}$ emits a positron and a $1.157 \mathrm{MeV} \gamma$-ray in quasicoincidences. In this case, the position of the emission point in the patient can be localized by the following method: firstly, the detection of a pair of $511 \mathrm{keV} \gamma$-rays produced by the positron annihilation allows us to reconstruct the Line Of Response (LOR). Then, the third $\gamma$-ray interaction located in the active volume of the Compton camera helps us to define the Compton cone [3]. With the kinematics of the Compton scattering process, the opening angle $\theta$ and the axis $\Delta$ of this cone are deduced. Finally, the position of the radiopharmaceutical labeled with ${ }^{44} \mathrm{Sc}$ is localized by the intersection of the LOR and the reconstructed Compton cone [4], [5].

This novel imaging modality provides a possibility to reconstruct the position of the radiotracer directly at a relatively low counting rate under the circumstance of the requirement of a dedicated Compton camera with high sensitivity and a large Field Of View (FOV) [6], [7]. A Liquid Xenon Time Projection Chamber (LXeTPC), whose detection medium LXe has been widely used in the field of particle physics and astrophysics, represents a perfect option to achieve these requirements [8].

Although the long-term objective of the XEMIS project revolves around the development of a large FOV LXe Compton camera for low-dose whole human body imaging, the first prototype, called XEMIS1, has demonstrated the capacity of a LXeTPC in $3 \gamma$ imaging technique. The 
cylindrical detector with an active volume of $2.8 \mathrm{~cm} \times 2.8 \mathrm{~cm}$ $\times 6$ or $12 \mathrm{~cm}$ is filled with nearly $30 \mathrm{~kg}$ of ultra-high-purity LXe. The detector performance of XEMIS1 is characterized by using another $\left(\beta^{+}, \gamma\right)$ radionuclide, the sodium-22 $\left({ }^{22} \mathrm{Na}\right)$. XEMIS1 shows promising results with a roughly $100 \mu \mathrm{m}$ position resolution along the drift direction (z-axis) and a good energy resolution of $9 \%$ (FWHM) for $511 \mathrm{keV} \gamma$-rays under a drift field of $1 \mathrm{kV} / \mathrm{cm}$ [5], [9].

\section{XEMIS2 CAMERA}

For the purpose of exploiting the advantages of the $3 \gamma$ imaging modality, a larger-scale LXe Compton camera and relative cryogenic infrastructures for small animal medical imaging, called XEMIS2, is under construction. The XEMIS2 overall system involves the XEMIS2 cryostat, the cryogenic subsystem of Recovery and Storage of Xenon, called ReStoX, and the purification and re-circulation subsystems.

The XEMIS2 cryostat is a dedicated single-phase LXe Compton camera, which handles up to nearly $200 \mathrm{~kg}$ of ultrahigh-purity LXe. The use of LXe as a detection medium allows constructing monolithic and homogeneous detectors to maximize detection efficiency. Besides, the innovative geometry of the camera with a large axial FOV favoring a high detection sensitivity allows exploiting low-dose wholebody small animal imaging. The camera consists of two identical large-scale LXeTPC sharing the same cathode located at the center of the detector. Each of the cylindrical LXeTPC contains an active volume with a drift length of 12 $\mathrm{cm}$, an inner radius of $7 \mathrm{~cm}$, and an outer radius of $19 \mathrm{~cm}$. The design of the XEMIS2 camera is illustrated in Fig. 1.

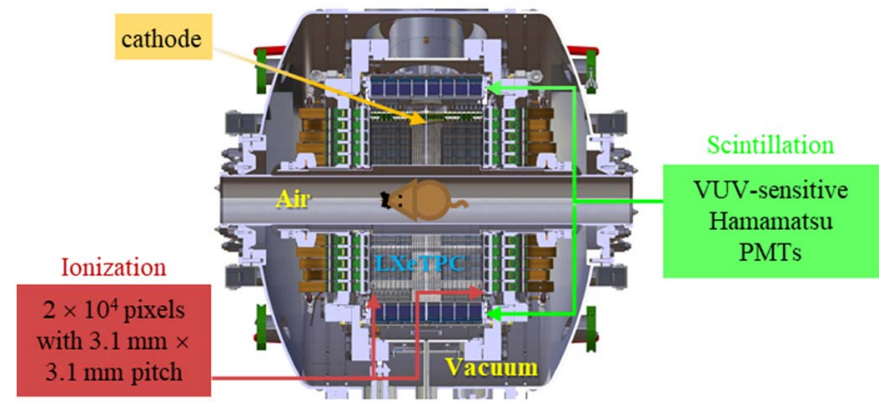

Fig. 1. Cutout view of the XEMIS2 camera for small animal imaging.

When a $\gamma$-ray emitted from the small animal interacts with the LXe in the detector, LXe has an essential feature of producing both scintillation light in the vacuum-ultraviolet (VUV) region and ionization charge carriers simultaneously. The VUV scintillation photons are detected by the VUVsensitive photomultiplier tube (PMT). Besides, the charge carriers are directly collected by the segmented anodes, which are shielded by the meshes used as Frisch grids. The camera is equipped with two pixelated anodes distributed on both sides of the detector, and each of them is segmented into 10000 pixels with a size of $3.175 \mathrm{~mm} \times 3.175 \mathrm{~mm}$. To drift the ionization electrons towards the pixelated anode in each of the cylindrical LXeTPC, a uniform electric drift field up to 2 $\mathrm{kV} / \mathrm{cm}$ is applied by an internal set of copper field shaping rings and an external set of stainless steel field shaping rings, which are placed along the z-axis. After collecting by the anodes, the ionization charge signals are treated by the dedicated electronics with ultra-low charges threshold level to extract the transverse $(\mathrm{X}, \mathrm{Y})$ coordinates and deposited energy per interaction vertex [5], [9].

An ultra-high purity level of the LXe is required for the XEMIS2 system since the VUV photons, or the electrons can be absorbed by some impurities dissolved in LXe, that degrade the scintillation light and attenuate the ionization charges. In order to realize and maintain an ultra-high purity level, a gaseous purification subsystem equipped with two rare-gas purifier getters and a re-circulation closed-loop containing the ReStoX and the oil-free membrane pump are equipped in XEMIS2 overall system realizing a continuous purification and circulation of the xenon. Before xenon enters the gaseous purification system, it is evaporated by a coaxial heat exchanger and then liquefied back in the cryostat [7].

Safe manipulation of a large quantity of xenon in a hospital center is achieved through the cryogenic subsystem ReStoX which has been successfully commissioned [10]. XEMIS2 cryostat and the cryogenic subsystem ReStoX have already been installed in a small animal medical imaging center CIMA at Nantes Centre Hospitalier Universitaire (CHU), as illustrated in Fig. 2. XEMIS2 is recently under construction.

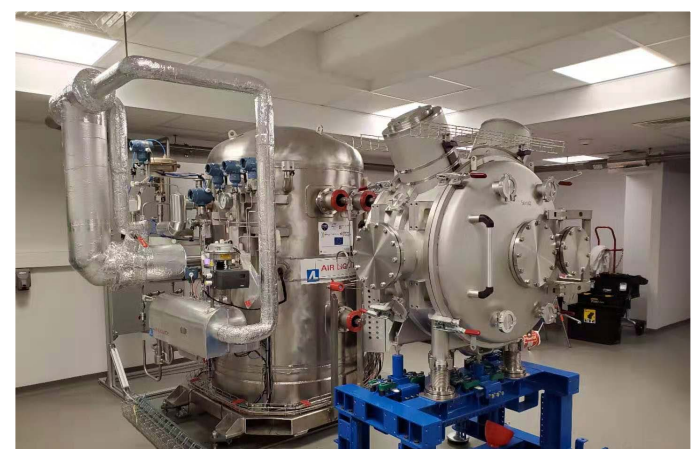

Fig. 2. Installation of the XEMIS2 cryostat and the cryogenic subsystem ReStoX in a small animal medical imaging center CIMA at Nantes Centre Hospitalier Universitaire (CHU).

\section{SCINTILLATION Light MEASUREMENT}

In the first stage, 64 PMTs are placed around the surface of the active area of the XEMIS2 camera for the sake of measuring the VUV scintillation photons. This configuration aims at showing the possibility of realizing whole-body small animal imaging with only $20 \mathrm{kBq}$ administered activities, for an exposure time of 20 minutes. In a second stage, 380 PMTs will completely cover the active volume of XEMIS2 in the context of increasing the injected activity to shorten the exposure time [11].

The VUV scintillation photon emission in LXe is a fast process, with an average lifetime of about $30 \mathrm{~ns}$ at a drift field of $2 \mathrm{kV} / \mathrm{cm}$. This feature makes LXe suitable for timing applications. In this case, the interaction time $\mathrm{t}_{0}$ of an ionizing particle (such as a $\gamma$-ray) with LXe is provided by the measurement of the scintillation light. While electron 
collection by the segmented anode gives the transverse $x-y$ position of each interaction, it is possible to associate the scintillation signals with the ionization signals to achieve the reconstruction of the interaction depth (i.e., the longitudinal zposition) with a resolution of $100 \mu \mathrm{m}$, which is calculated by the product of the electron drift time, referred to the $\gamma$-ray interaction time $t_{0}$, and the electron drift velocity depending on the applied electric drift field. In order to achieve such accuracy, a scintillation light detection chain with a time resolution below $50 \mathrm{~ns}$ is required.

Furthermore, it is possible to pre-localize the $\gamma$-rays interactions spatially and then achieve the virtual fiducialization of the active volume by matching the scintillation signals with the ionization signals through the event builder. In the context of increasing the injected activity to shorten the exposure time, the spatial pre-localization of $\gamma$ ray interactions contributes to the reduction of the occupancy in the LXeTPC caused by the nonnegligible electrons drift time up to $60 \mu \mathrm{s}$.

To carry out a continuous $(100 \mathrm{MHz})$ data taking with negligible electronics dead-time, a new scalable data acquisition (DAQ) system has been specially developed for XEMIS2. This DAQ system contains two independent parts: scintillation light and ionization charges detection chains with time synchronization.

\section{A. Scintillation Light Detection Chain}

A novel scalable self-triggered scintillation light detection chain has been specially developed for XEMIS2 to measure the interaction time of a $\gamma$-ray with LXe and the number of scintillation photons produced after the interaction. This scintillation light measurement system includes four function units: VUV-sensitive PMT, pulse-shaping amplifier, discriminator, and XEMIS Data Concentrator (XDC) [12].

In order to detect the scintillation light with an emission wavelength of $178 \mathrm{~nm}$, we select the VUV-sensitive Hamamatsu R7600-06MOD-ASSY PMT, which is specially designed to withstand the temperature of LXe [11].

A dedicated pulse-shaping amplifier is developed to filter, shape, and amplify the output signals of the PMT. For a more detailed description of the pulse-shaping amplifier, please refer to [11], [12]. The scintillation light in LXe is characterized by three different decay components under a drift field of $2 \mathrm{kV} / \mathrm{cm}$ : $3 \%$ fast component (i.e., the singlet excited state) with a characteristic time constant of $2.2 \mathrm{~ns}$ [13], $60 \%$ slow component (i.e., the triplet excited state) with a decay time of $27 \mathrm{~ns}$ [13] and 37\% third component due to electron-ion recombination with a lifetime of 45 ns [14]. Generally, the pulse duration of PMT is about $50 \mathrm{~ns}$ in XEMIS1, as illustrated in Fig. 3. Then, the PMT output signals are effectively integrated and amplified by the pulseshaping amplifier, with about $50 \mathrm{~ns}$ peaking time, as illustrated in Fig. 4. The choice of the parameters of the pulse-shaping amplifier is a good compromise between fully integrating the signal and reducing the pulse pile-up.

There is no global event trigger for data acquisition in XEMIS2. Each PMT channel is self-triggered by adding a leading-edge discriminator with a constant threshold voltage acting on the shaped pulse. Since the scintillation signals are not used to measure precisely the deposit energy in XEMIS2, the Time-Over-Threshold (TOT) method is used to determine approximately the number of photoelectrons (pe) collected by the VUV-sensitive PMT.



Fig. 3. Example of output signal of the photomultiplier tube (PMT).

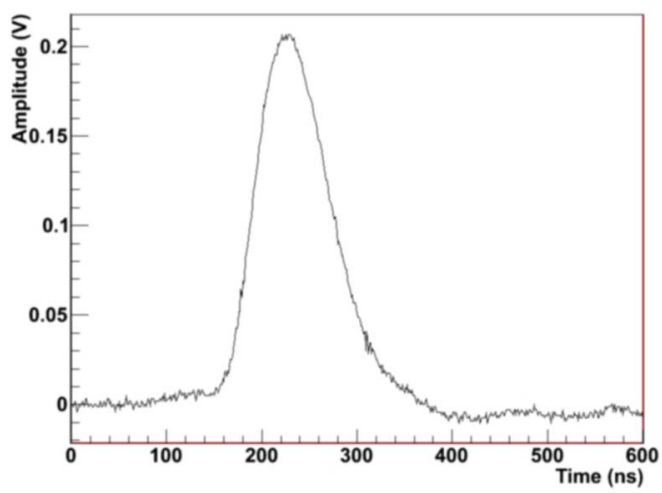

Fig. 4. Example of output signal of the pulse-shaping amplifier.

In order to perform efficient and convenient data processing, a low-cost 16-channels circuit composed of pulseshaping amplifiers and discriminators has been specially developed for XEMIS2. The analog signals from the PMTs are processed via this new scintillation light electronics, for the purpose of extracting the leading edges and trailing edges of the shaped pulses from each channel. A low threshold is applied to measure the signal corresponding to a few photoelectrons.

The XDC contains several Field-Programmable Gate Array (FPGA) components. The leading edge, trailing edge, and PMT address information from each channel are read out and digitized by the FPGA. Besides, the scintillation and ionization signal detection chains are synchronized by the XDC.

\section{B. Calibration of PMT gain}

Two VUV-sensitive PMTs have been currently calibrated in the XEMIS1 under the XEMIS2 operation condition to characterize the gain of PMT. In the calibration test, two PMTs located beside the TPC are entirely immersed in the LXe. The PMTs are illuminated by a very low light intensity 
LED coupled to an optical fiber that can extend to the inner of the camera. The waveform generator was connected to the 250 $\mathrm{MHz}$ Flash Analog-to-Digital Converter (FADC) for triggering purposes. This experimental setup permits us to measure and monitor the PMT gain during the operation. The experimental results demonstrate that the gain of PMT increases exponentially as the high-voltage of the PMT increases, as expected. Furthermore, the gain of PMT is almost stable at 1.2 bar and $168 \mathrm{~K}$ during the operation. The daily measurement of the PMT gain is illustrated in Fig. 5.

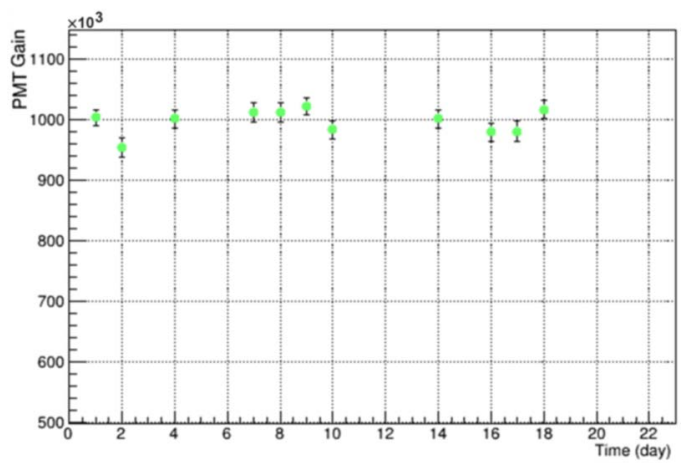

Fig. 5. Daily measurement of the PMT gain in XEMIS1.

\section{Calibration of scintillation light read-out electronics}

The scintillation light read-out electronics is currently calibrated in the XEMIS1 under the XEMIS2 operation condition to characterize the performances of scintillation light measurement. The experimental results showed that for each PMT channel, the pulse-shaping amplifier has a good electronic linearity response and quite similar behavior. The electronic noise of each channel of the read-out electronics is quite uniform and low. Besides, there is a correlation between the number of pe and the shaped pulse duration from the leading edge to the trailing edge (i.e., the TOT), as illustrated in Fig. 6. The results indicate that the number of detected pe can be easily measurable under the situation of collecting a few pe (very probably situation in XEMIS2). The intrinsic time resolution of scintillation light read-out electronics is less than $10 \mathrm{~ns}$, which is limited by the sampling frequency of FPGA of about $100 \mathrm{MHz}$.



Fig. 6. Correlation between the number of photoelectrons (pe) and the Time-Over-Threshold (TOT) with a threshold of 2 pe.

\section{CONCLUSION}

XEMIS2 combines the $3 \gamma$ medical imaging modality with the larger-scale LXe Compton camera, which represents a good option to realize low radiation dose whole-body small animal imaging. In XEMIS2, the scintillation signals provide the $\gamma$-rays interaction time. Furthermore, it is possible to prelocalize the $\gamma$-rays interactions spatially and then achieve the virtual fiducialization of the active volume by matching the scintillation signals with the ionization signals. A selftriggered scintillation light detection chain has been specially developed for XEMIS2 to carry out continuous data taking with negligible electronics dead-time. It is currently calibrated in the prototype XEMIS1. The calibration results indicate that the dedicated detection chain has a good performance in scintillation light measurement at the first attempt. XEMIS2 is recently under construction in a small animal medical imaging center CIMA at Nantes Centre Hospitalier Universitaire (CHU) for further preclinical studies. Besides, XEMIS2 is an important preliminary step towards the development of a low radiation dose whole human body imaging system, XEMIS3.

\section{REFERENCES}

[1] C. Grignon et al., "Nuclear medical imaging using $\beta+\gamma$ coincidences from Sc-44 radio-nuclide with liquid xenon as detector medium," Nucl. Instrum. Meth. A, vol. 571, 2007, pp. 142-145.

[2] S. Huclier-Markai et al., "Promising Scandium Radionuclides for Nuclear Medicine: A Review on the Production and Chemistry up to In Vivo Proofs of Concept," Cancer biotherapy \& radiopharmaceuticals, vol. 33, no. 8, 2018, pp. 316-329.

[3] D. B. Everett, J. S. Fleming, R. W. Todd, and J. Nightingale, "Gammaradiation imaging system based on the Compton effect," Proc.IEEE, vol. 124, 1977, pp. 995-1000.

[4] T. Oger et al., "A liquid xenon TPC for a medical imaging Compton telescope," Nucl. Instrum. Meth. A, vol. 695, 2012, pp.125-128.

[5] L. Gallego Manzano et al., "XEMIS: A liquid xenon detector for medical imaging," Nuclear Instruments and Methods in Physics Research A, vol. 787, 2015, pp. 89-93.

[6] J. P. Cussonneau et al., "3 $\gamma$ Medical Imaging with a Liquid Xenon Compton Camera and ${ }^{44}$ Sc Radionuclide," Acta Phys. Pol. B, vol. 48, no. 10, pp. 1661, Oct. 2017.

[7] L. Gallego Manzano et al., "XEMIS2: A liquid xenon detector for small animal medical imaging," Nucl. Instrum. Meth. A, vol. 912, 2018, pp. 329-332.

[8] E. Aprile, and T. Doke, "Liquid Xenon Detectors for Particle Physics and Astrophysics," Rev. Mod. Phys., vol. 82, no. 3, 2010, pp. 20532097.

[9] Y. Xing et al., "XEMIS: Liquid Xenon Compton Camera for $3 \gamma$ Imaging," 4th International Conference on Technology and Instrumentation in Particle Physics (TIPP), Springer Proc. Phys., vol. 213, 2017, pp. 154-158.

[10] L. Virone et al., "Gravity assisted recovery of liquid xenon at large mass flow rates," Nucl. Instrum. Meth. A, vol. 893, 2018, pp. 10-14.

[11] Y. Zhu et al., "Scintillation Signal in XEMIS2, a Liquid Xenon Compton Camera with $3 \gamma$ Imaging Technique," 4th International Conference on Technology and Instrumentation in Particle Physics (TIPP), Springer Proc. Phys., vol. 213, 2017, pp. 159-163.

[12] Y. Zhu et al., "XEMIS2: A liquid xenon Compton camera to image small animals," 2019 IEEE $20^{\text {th }}$ International Conference on Dielectric Liquids (ICDL), Roma, Italy, 2019, pp. 1-4.

[13] T. Doke, "Fundamental properties of liquid Argon, Krypton and Xenon as radiation detector media," Portugal. Phys., vol. 12, 1981, pp. 9-48.

[14] S. Kubota, M. Hishida, M. Suzuki, and J.Z.R. Gen, "Dynamical behavior of free electrons in the recombination process in liquid argon," krypton, and xenon, Physical Review B, vol. 20, no. 8, 1979, pp. 34863496. 